\title{
LA EVALUACIÓN ACADÉMICA DE LOS PROYECTOS DE DISEÑO EN LOS TALLERES DE DISEÑO INDUSTRIAL
}

\author{
Abdénago Yate Arévalo \\ Estudiante de Doctorado en Bioética \\ Magíster en Investigación en Problemas Sociales Contemporáneos \\ Diseñador Industrial. Estudiante de Filosofía \\ abdenagoyate@gmail.com
}

Recibido: 18-12-2014, aprobado: 09-02-2016, última versión: 09-02-2016¹

\section{RESUMEN}

El presente artículo es resultado del proyecto de investigación "Modelo de evaluación en el proceso de aprendizaje-enseñanza de los proyectos de diseño en los talleres de diseño" realizado al interior del grupo de investigación Polisemia Digital, categoría D en Colciencias. En torno a un análisis teórico crítico reflexivo se generó un modelo de evaluación al cual se le crearon herramientas que permiten la evaluación académica de los proyectos de taller de diseño, y por medio de pruebas piloto, en diferentes talleres, proyectos y semestres durante tres años de trabajo, se logra la comprobación de la aplicación de dichas herramientas con el fin de obtener una realimentación del proyecto. Así, se muestra la herramienta de evaluación, su estrategia de aplicación, los criterios valorados y las estadísticas respectivas, que permiten un seguimiento para mejorar los procesos llevados a cabo al interior de los talleres de diseño. De igual manera, se presentan conclusiones, bajo la modalidad de sugerencias, para el mejoramiento de los procesos tanto de la investigación como de los métodos de aprendizajeenseñanza desde el enfoque evaluativo de las áreas del diseño, ya que la investigación evidencia las similitudes en modelos pragmáticos pedagógicos y evaluativos de las distintas disciplinas de diseño.

Palabras clave: Evaluación, Diseño Industrial, Herramientas, Academia, Taller de Diseño.

\section{Abstract}

This article is the result of the research project "Model evaluation in the teaching-learning process of design projects in the design workshops" conducted within the research group Polisemia Digital, category D Colciencias. Around a theoretical analysis generated a critical reflective assessment model which was created tools that enable the academic evaluation of the design studio projects, and through pilot testing in various workshops, projects and semester for three years work, it is possible to check the application of the tools in order to obtain a feedback of the project. This shows the assessment tool, its implementation strategy, the criteria evaluated and the respective statistics, which allow monitoring to improve the processes carried out within the design workshops. Similarly, conclusions, in the form of suggestions for the improvement of both research processes and methods of teaching-learning from the evaluation approach in the areas of design, research and evidence that the similarities in pedagogical and evaluative pragmatic models of different design disciplines.

Keywords: Evaluation, Industrial Design, Tools, Academia, Design Workshop.

El artículo es resultado del proyecto interno de investigación "Reflexión crítica de la teoría del diseño industrial", desarrollado en el grupo de investigación adscrito a la Universidad Autónoma de Colombia, Polisemia Digital, categoría D en Colciencias, en su línea de investigación de Teoría de Diseño. 


\section{INTRODUCCIÓN}

...la evaluación debe facilitar la posibilidad de planear, transformar, modelar, definir y hacer un seguimiento de las prácticas pedagógicas, permitiéndonos conocer lo que sucede no sólo en el aula de clase, sino que prevé los impactos e incidencias que éstas tienen en el aprendizaje del estudiante. (Yate A. et al., 2010:68).

Conceptos que tienen gran influencia en la academia y en el mercado son: disciplinas y escuelas de diseño... enseñanza y aprendizaje... profesión y productos... evaluación. Este último -que realmente serían dos: a nivel académico y a nivel pragmático dentro del contexto empresarial- de los elementos que constituye la práctica del diseño en sus diferentes disciplinas por cuanto se juega con el parámetro de la integralidad para involucrar los aspectos: humano, empresarial, social y académico. Sin olvidar que la integralidad desde el conocimiento de una disciplina, se envuelve en un sinnúmero de aspectos, sin embargo en este texto se considerarán baladí, a pesar de lo cual serán tenidos en cuenta de manera superficial y/o tangencial. No obstante, vale la pena mencionar que los fundamentos que deberán ser contemplados en el momento de evaluar un diseño -o proceso de diseño-, son determinados por la filosofía y el perfil profesional que pretende potenciar hacia la sociedad las escuelas de diseño, debido a que sus fortalezas y sus debilidades son comprometidas a sus modelos de enseñanza.

Discursos y retóricas metodológicos que son propuestas a niveles teóricos, se encuentran hábilmente expuestas, pero a niveles sofistas, que pretenden contemplar factores integradores del proceso de evaluación. Sin embargo, la sensatez deberá permitir encontrar la dificultad nativas a su vez reflexivas, para solventar las grandes necesidades de contar con una -o varias preferiblemente- solución para hacer estoico el proceso evaluativo en las disciplinas de diseño, que por su composición: ciencia-tecnología-arte, cuya escisión ha sido causada por el ortodoxo planteamiento disciplinar de los requerimientos de algunos pocos, en tanto que dificultan la integralidad del ser humano, más allá de la poïesis, hasta dominar el diseño.

La fortuna disciplinar se encuentra cercana hacia su asimilación como discurso compuesto por la trilogía anteriormente expuesta, debido a que se considera la seriedad de la temática dentro del proceso de aprendizaje/enseñanza -es bien sabido que se le ha denominado enseñanza/aprendizaje, mas se invierte debido a la jerarquía del autoaprendizaje-; por cuanto doy paso a la reflexión: ¿cómo evaluar diseño?

\subsection{Factores incidentes en el proceso}

Los principales factores que inciden en el proceso evaluativo de diseño: educación, aprendizaje, ser humano y normas, todos ellos transversalizados por la cultura como componente infalible e indispensable en todo contexto humano, abordados de forma integral reflejan la necesidad de contemplar en la evaluación de diseño parámetros que contemplen la cultura, la sociedad, la empresa, la academia y el factor humano, que a su vez se interrelacionan en el Proceso-Resultado.

Existe un elemento que ha sido escindido de la academia desde el punto de vista del desarrollo de diseño: el factor costo, es decir el componente económico, desde el punto de la medición de costo/beneficio en cuanto al resultado propuesto y su potencial efectividad en el mercado. Su peso radica en la alienación que conlleva mentes de solución de necesidades y satisfacción de deseos por medio del plusvalor que otorga el factor económico. Así, intrapersonalmente subyacen los valores y principios para satisfacer los deseos, que serán reflejados 
de una u otra forma, en diseño -o por lo menos en propuesta-, por cuanto se hace necesario el entendimiento del componente cultural, como indispensable en el análisis, para su posterior potencialización, de las propuestas de diseño por parte de los aprendices, que pretenden positivar sus propuestas con una alta carga de contenido de deseo subjetivado.

Algún otro deducible, es la necesidad de paralelizar el proceso de evaluación, con el proceso de aprendizaje/enseñanza, es decir, la constante evaluación -con todas sus variantes: co, inter, intra, hetero... y todas aquellas que sean subdivisiones- (realimentación evaluativa) en el discurrir del proceso de diseño, que requiere un seguimiento continuo por parte de los versados en el tema. La frase precedente, nos lleva a una nueva reflexión: Más allá de las disposición de los docentes, en cuanto al proceso evaluativo, debe ser entendido que el diseño -al igual que el universo de las distintas disciplinas- está compuesto por una gran variedad de disciplinas que la hacen ser, por cuanto la capacitación de los evaluadores debe ser a nivel inter y transdisciplinar; a su vez se debe entablar una relación dialéctica, que determine la efectividad de la enseñanza impartida para con los conocimientos adquiridos en el proceso.

\subsection{El Pentágono con Eje Radial}

A continuación se presenta un sistema de cuestionamiento que coadyuva en la reflexión que se fecunda en el ser humano.

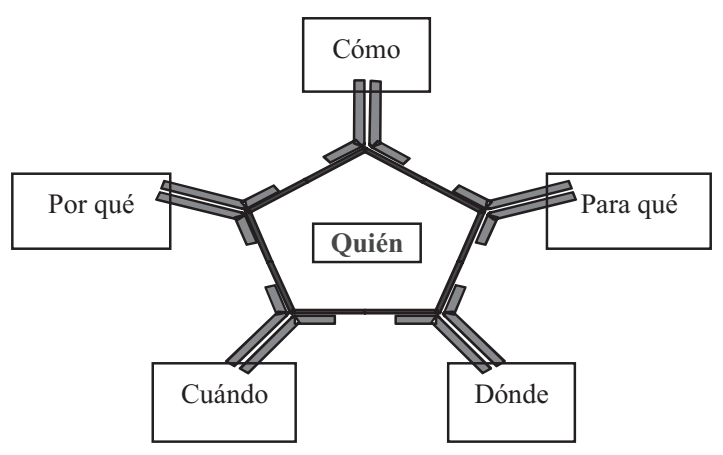

Gráfico 1

Pentágono de cuestionamiento evaluativo
A partir del análisis de los componentes anteriores, se logra vislumbrar el alto contenido de significación e importancia que hace que el ser humano sea el centro de los procesos evaluativos que componen la aprehensión y actuación de diseño, siempre caracterizada en el contexto socio/cultural -espacio/temporal- que hace el reconocimiento de la efectividad (Pertinente) del producto final.

No es baladí de esta manera, los métodos implementados en el proceso de aprendizaje/enseñanza de diseño, para que sean una manguala sinérgica, en la que dicha simbiosis lleva a una formación reflexiva e integral, que permitan una equidad en los resultados evaluativos de índole cualitativos y cuantitativos, con lo cual se logra elevar los niveles de competencia de los diseñadores (personas/profesionales) que respondan tanto a las necesidades personales, como a las socio-empresariales.

\section{La eVALUACIÓN ACADÉmica DISCIPLINAR EN DISEÑO INDUSTRIAL}

Es sabido las amplias reflexiones que han tenido lugar a lo largo de la historia los conceptos de disciplina, disciplinariedad, interdisciplinariedad y transdisciplinariedad, por cuanto daremos por hecho la preexistencia de las estructuras semánticas de los mismos ${ }^{2}$, entendiendo: disciplina es el campo de acción ${ }^{3}$ de los conocimientos de un área del saber determinada a niveles instauradas en un contexto socio-comercial; para ejemplos prácticos las disciplinas del diseño gráfico, diseño industrial, las artes, entre

2 Baladí el discurso sobre la asertividad de los términos, debido es a que lo que se pretende es reflexionar en cuanto a la necesidad de una interrelación equitativa de los tres conceptos, por cuanto dejamos en libre albedrío la determinación de sus aportes a sus estructuras semánticas, las cuales en pro del proceso cognitivo, esperamos se susciten inquietudes.

3 Campo de acción hace referencia a la sinergia espaciotemporal tanto en la aprehensión del conocimiento, la aplicación de los mismos, al igual de la formulación de propuestas para su crecimiento. 
otras; la interdisciplinariedad conjuga la relación sinérgica entre disciplinas en uno o varios campos de acción del conocimiento; la transdisciplinariedad conlleva al rompimiento de los límites de los campos de acción de una disciplina, instaurando un solapamiento del conocimiento por parte de otra disciplina en la cual el constructo cognitivo es asinérgico.

A la luz de planteamientos precedentes se pretende a continuación dar pie y base a la necesidad de la implementación tanto de la disciplinariedad, la interdisciplinariedad y la transdisciplinariedad en los procesos de enseñanza-aprendizaje del diseño industrial.

Las academias acertadamente presentan un conjunto y repertorio de conocimientos que ponen a disposición de los estudiantes para que ellos aprendan, especulen y propongan al interior de una disciplina específica, con lo cual se busca una apropiación de conocimientos de orden fáctico (en especial las instituciones técnicas y tecnológicas), con los que ellos pueden hacer frente al contexto, dentro de los parámetros pragmáticos, y más aún dentro de los imaginarios socio-culturales y económicopolíticos en los cuales el "título" que posea una persona, la "etiqueta disciplinar" permite un reconocimiento de la especialización en conocimientos aprehendidos; se recalca esta frase ya que ella permite hacer un nuevo derrotero en el proceso cognitivo, dado por el contexto industrial, donde entra a desempeñar un papel muy importante la interdisciplinariedad.

Cuando se habla de un conocimiento impartido-aprendido en la academia, y observamos los pénsum, los currículos, los programas de las asignaturas y lo sumamos al contexto tanto 70 abre el abanico de los conocimientos disciplinares y se convierte en conocimientos interdisciplinares, ya que la naturaleza disciplinar fue construida a partir de conocimientos adquiri- cimiento pragmático. Tomemos como ejemplo el diseño industrial, ya que es encuentra como objeto del presente texto.

El diseño industrial para el dominio de la expresión hace uso del lenguaje formal (dominio de la estética), de un conocimiento de un mercado objetivo o "target Market" (dominio de la mercadotecnia), de los criterios de percepción de las personas (dominio de la psicología), de materiales y procesos (dominio de las ingenierías), entre otros así podríamos enumerar más elementos cognitivos de otras disciplinas ${ }^{4}$, sin embargo sería baladí hacerlo. Ahora bien, no es menester el aprendizaje erudito del conocimiento universal, ya que lo que pretende una disciplina de las otras, es la aprehensión del conocimiento en pro de su propio fortalecimiento, tanto de orden teórico como práctico, es así como se ha estado enseñando-aprendiendo las disciplinas, algo que se pretende desconocer debido al dogmatismo imperante; veamos pues que este solapamiento cognitivo presenta características sinérgicas, que gracias a las solicitudes del medio ha dado lugar a disciplinas como la electromedicina, la electromecánica, la ecología social, la ingeniería de mercados ${ }^{5}$, entre otras.

Estos constructos multidisciplinares, son de manera sistemática, organizada, pensada, racionalizada. No obstante, el contexto industrial y económico, a pesar de su camino a la microespecialización, tiene la otra cara de la moneda, en la cual se solicita personal lo suficientemente calificado en la versatilidad de trasgresión en una disciplina y otra, debido a la disminución

4 No es en este texto la intención de encontrar los criterios disciplinares del diseño industrial, la intención es reflexionar sobre la construcción de su conocimiento de aplicación. dos, propios y ajenos, que permitan su fortale-
La ingeniería de mercados, sin ahondar en el tema, cuenta con características de interdisciplinariedad similares a los diseños gráfico e industrial, con su gran fortaleza en el marketing. Algo que sugiero al lector, no ser ajeno a la construcción de disciplinas multidisciplinares. 
de costos en mano de obra, tiempo y espacio, por cuanto se estructuran relaciones asinérgicas entre las disciplinas, creando la transdisciplinariedad por solicitud (probablemente no) expresa de la socio-economía. Por esta razón no debemos ser apáticos a esta realidad.

Ser coercitivo en las exploraciones transdisciplinares, disminuyen la capacidad en creatividad y versatilidad de las personas, en especial de los estudiantes, aunque debe entenderse que el docente es un guía, no un dictador asincrónico de la realidad emergente.

A manera de una reflexión en aproximación concluyente ¿Es o no necesario reevaluar la inequidad existente en la academia de la relación disciplinariedad - interdisciplinariedad transdisciplinariedad? Debido a que la castración sistemática por los procesos de formación de los conocimientos adquiridos asincronizan a los estudiantes con su contexto, colocando en un extremo de la cuerda la investigación y en otros la pedagogía tradicional y ortodoxa de la inequidad en dicha relación.

\section{Principios (Reflexiones) de la Pedagogía (Andragogía) 6 POSTMODERNA}

En los principios de la pedagogía postmoderna (PPM) no es indiferente la noción de la responsabilidad del estudiante para con su proceso de aprendizaje, la versatilidad del docente presto a los cambios psicodinámicos en dicho proceso; por cuanto los entes dinamizadores primarios del conocimiento fuera y dentro del aula respecto a una profesión y/o disciplina (los estudiantes y los docentes) deben encontrar un equilibrio entre la estructura académica y

6 En el caso que se presenta referente a la diferenciación entre pedagogía y andragogía, etimológicamente hablando, que tiene repercusiones en el proceso cognitivo de aprendizaje-enseñanza, se tendrá en cuenta la palabra pedagogía para denotar y connotar a ambas. los cambios del contexto socio-económicos que representan.

De esta manera, la responsabilidad sobrepasa el status quo, respecto al estado del estudiante receptivo de información y en el mejor de los casos recopilador de síntesis externas (ajenas) para balbucear síntesis propias, para la búsqueda de proposiciones que no sólo permitan virtualizar el pensamiento hacia reflexiones temporales, sino que conlleven a nuevos derroteros de acción que abren el abanico de opciones pragmáticas para el logro de los objetivos de los proyecto, asignatura, semestre, currículo, profesión o disciplina; de esta manera se logra la construcción de propuestas de orden práctico en la enseñanza, que permitirán una evolución en los procesos pedagógicos.

Para ello es menester, entender al docente con la capacidad no sólo receptiva de las propuestas, sino un sintetizador y catapultador de materialización de ideas, por cuanto requiere una evolución y replanteamiento constante tanto a nivel pedagógico académico como socio-cultural siendo consecuente con el medio en el cual se desenvuelve la disciplina: académica y profesionalmente. Si las exigencias del medio van evolucionando, cambiando, tomando nuevos caminos, al igual que el pensamiento humano, inquieto, versátil, crítico, constructivo (huelga comprender que una disciplina no es estática), ella cambia para acoplarse o proponer alternativas, desarrollos, por cuanto no se haría justicia por medio de la estaticidad académica y pedagógica que se relega, y debilita la disciplina y a sus profesionales.

Muchos son los discursos académicos que se entablan en un sinnúmero de textos, cursos y discursos, referidos a los procesos de aprendizaje-enseñanza, desbordan la creatividad en sus planteamientos, sistematizan el proceso, jerarquizan los indicadores, ponderan las actuaciones, categorizan los actores y dan pautas de orden correctivas y preventivas, que recaen 
en los hombros del docente, automatizándolo, proponiendo su robotización comportamentacional, y en las entidades de ecuación superior damos culpas a la educación media, ellos a su vez a la educación básica, ellos a los hogares, ellos a la sociedad, que a su vez otorgan su responsabilidad a las entidades de educación superior, todos se lavan las manos de manera magistralmente demagógica, bajo retóricas muy elaboradas, sobre inmensos y bien articulados sofismas, pero que todas se encuentran fuera de las manos de las personas físicas, y se encuentran dentro de los límites de entes virtuales, preocupados siempre en enseñar el saber-saber, en el saber-hacer, debido al pensamiento, absurdo a mi manera de ver, de no relacionar el saberser, por no rozar con la moralidad, la ética, lo personal, lo religioso, lo humano, lavándose las manos creando asignaturas como ética profesional, convirtiendo el saber-ser en un algo del sistema, colgada como un apéndice inevaluable, problemático y "jarto". Un ejercicio analógico reflexivo es: el lenguaje es producto del proceso cognitivo como medio socializador para la transmisión de conocimientos. La responsabilidad y el saber-ser es un producto social derivado de la transmisión de conocimientos, por medio del lenguaje como socializador, cabe la pregunta ¿si la academia permite la transmisión de los conocimientos productos de la interrelación social, los lenguajes disciplinares como componente del saber-saber y el saber-hacer se encuentra al mismo nivel de la responsabilidad como componentes del sabersaber y el saber-hacer en cuanto configurado del saber-ser?

Las disposiciones, en tanto que hasta el momento han sido de orden impositivas o permisivas, en sus dos polos, permiten la corrupuna búsqueda trilógica entre el saber-saber, el sus decisiones y entorno; por cuanto la pedagogía postmoderna no plantea el desvirtuamiento de los conceptos que sustentan y desarrollan el saber-saber y el saber-hacer, sino que hace saber-hacer y el saber-ser, a la luz de la sociodinámica, por cuanto las identidades individuales y sociales se entremezclan en emergencias contextuales.

\section{Preparación de los estudiantes HACIA LA INVESTIGACIÓN}

El boom que se ha suscitado respecto a la necesidad de involucrar la investigación en los procesos académicos han llevado a una creciente demanda referida a capacitaciones en métodos de enseñanza de todo los docentes en el sinnúmero de disciplinas que componen las instituciones académicas actuales, dando paso a procesos pedagógicos flexibles en cuanto a traspaso de conocimientos, algunos realimentados y otros propositivos, que dan un nuevo valor al sentido de pertenencia y compromiso para con los procesos cognitivos. Sin embargo, esta amplia gama pedagógica se desvirtúa frente a la inflexibilidad de las planillas, cuadros y recuadros, formatos, y el abanico excepcional de normativas administrativas que de manera a priori aprisionan todos los cambios que se suscitan mediante la sociodinámica tanto a niveles individuales como grupales que logran permear el proceso de aprehensión del conocimiento.

El conocimiento de manera espectacular y abrumadora, ha demostrado su movilidad constante, su evolución, su continuo crecimiento sobre diferentes caminos, tanto generales como especializados, lo que lleva al cuestionamiento ¿por qué la academia se empeña en que las temáticas y los proyectos, sean estáticos?, y más aún ¿qué se está haciendo para cambiar el pensamiento de que el conocimiento al interior de una asignatura debe ser estático sobre el pretexto de ser la bases del concepto estudiado?

La actualización del conocimiento, es bien sabido, pero ignorado, es necesario hacerlo en cada una de las asignaturas, debido a la necesidad que impone el mismo conocimiento como 
los niveles prácticos que son impuestos por el medio socio-cultural y político-económico.

Cuando se entremezclan los procesos académicos y los investigativos, es menester el entendimiento de desarrollar programas, sean estos curriculares o de asignaturas, que permitan la formulación de cuestionamientos, proposiciones y alternativas en generación de conocimiento que den al estudiante las herramientas para la construcción de nuevo conocimiento, que permita trascender el método tradicional de enseñanza, en el cual el docente plantea su asignatura bajo sus propias premisas sin constatar las exigencias del medio, la cultura de los grupos, y la evolución del conocimiento, entablando así la adinamización del proceso cognitivo.

Es importante que los parámetros establecidos para el proceso de educación, se encuentren en una constante evaluación y re-evaluación, que permita la aprehensión del conocimiento acordes al medio y a las personas.

\section{SinERGIA CURRICULAR: RELACIONES FÁCTICAS DE LAS ASIGNATURAS VINCULADAS}

Cuando se habla de la vinculación sistémica de las asignaturas de un programa curricular con el fin de lograr objetivos respecto a una profesión o disciplina, se entablan relaciones en tanto la existencia de una asignatura como eje integrador que subordina las otras asignaturas en desmedro de sus potencialidades, ya que el dominio de unos objetivos específicos del eje pervierten los objetivos específicos de sus coadyuvantes disciplinares, por varias causas entre las que se encuentran: la subjetivación del conocimiento por parte del docente encargado del eje integrador; el planteamiento de proyectos discordes con las necesidades del medio, la falta de análisis respecto a la formulación del tiempo que se debe dedicar a las temáticas disciplinares, interdisciplinares y transdisciplina- res, el planteamiento respecto a la búsqueda de cumplir los objetivos de la asignatura de eje integradora y no los objetivos temáticos disciplinares; entre otras que serán cuestionamientos intrínsecos al discurso planteado.

A la luz de estos parámetros discrónicos de los procesos de aprehensión del conocimiento, se generan relaciones parasitarias, en la cual el eje integrador se nutre del resto de las asignaturas en desmedro de ellas, las cuales se convierten en apéndices subordinados que redunda en la pérdida de su propia identidad, su virtuosismo temático y sus contribuciones propositivas para con la disciplina.

Sin lugar a duda, la relación entre las asignaturas debe existir, sin embargo, se debe plantear sobre la premisa de la sinergia curricular, en la cual la búsqueda de los objetivos en pro del fortalecimiento disciplinar sea dado por el sistema en comunión. Para ello se deben entablar proyectos cuyo génesis se encuentren en las temáticas intra, inter y transdisciplinares, y no a consecuencia de la asignatura de eje integrador.

Así, cabe anotar que la relación de las asignaturas no debe entablarse solamente en los momentos de formulación y conclusión del proyecto, ya que esto restringiría la evolución conjunta, por tanto se debe contar con canales de comunicación constante que permita la transmisión de conocimientos en todo momento para el afianzamiento, reevaluación y seguimiento del proyecto temático.

\subsection{La colegiada como mecanismo integrador}

En vista de la urgente necesidad de mecanismos integradores para la sinergia curricular en cuanto a sus componentes se refiere, la heteroevaluación o colegiadas es un elemento que fortalece el proceso cognitivo. La colegiada se presenta en forma de proyecto con carácter 
evaluativo de los contenidos o conocimientos asimilados y procesados en cada una de las asignaturas que se interrelacionan durante un periodo lectivo; ella debe contar con parámetros y reglamentos claros respecto a su finalidad, su proceso de desarrollo y su asertividad temática. En la colegiada se entiende que cada una de las asignaturas, sea ésta o no el núcleo problemático, deben apuntar a un objetivo común en cuanto a la aprehensión del conocimiento, para ello hay que tener en cuenta que si el objetivo se centra en los alcances de una sola asignatura, las demás se verán subordinadas, e incluso algunas serán forzadas, convirtiéndose en apéndices que desvirtúan las fortalezas y posibles logros de la colegiada.

Para el logro efectivo de la colegiada es necesario que programa curricular cuente con un objetivo claro del cual nazca el objetivo del semestre y de este a su vez el objetivo de cada asignatura que dará pie para la creación de los objetivos de la colegiada. A la luz de esto se vislumbra que los proyectos de colegiada deben estar determi- nados por unidades temáticas bajo la sinergia de las asignaturas, y no respecto a los deseos específicos de una asignatura en particular.

Surgen dos cuestionamientos a tener en cuenta: ¿Cuántas colegiadas son pertinentes durante un periodo lectivo? ¿Cómo es su articulación con proyectos particulares de cada asignatura?, para reflexionar sobre ello haremos uso de los procesos de evaluación de proyectos, pero estos serán extrapolados para hacer la evaluación no a un proyecto de colegiada, sino al grupo académico vinculado en los procesos de enseñanza-aprendizaje, a su vez que los tiempos de destinación, con dependencia en aplicación disciplinar con simulación en campo real de acción como ejes primarios. Vale la pena mencionar la existencia de parámetros que se sustentan a partir de ellos, como lo son: la presentación, el carácter, el equipo y la evaluación, sin embargo la programación de la colegiada con los parámetros primarios darán la génesis a los secundarios; que serán contemplados en páginas procedentes.

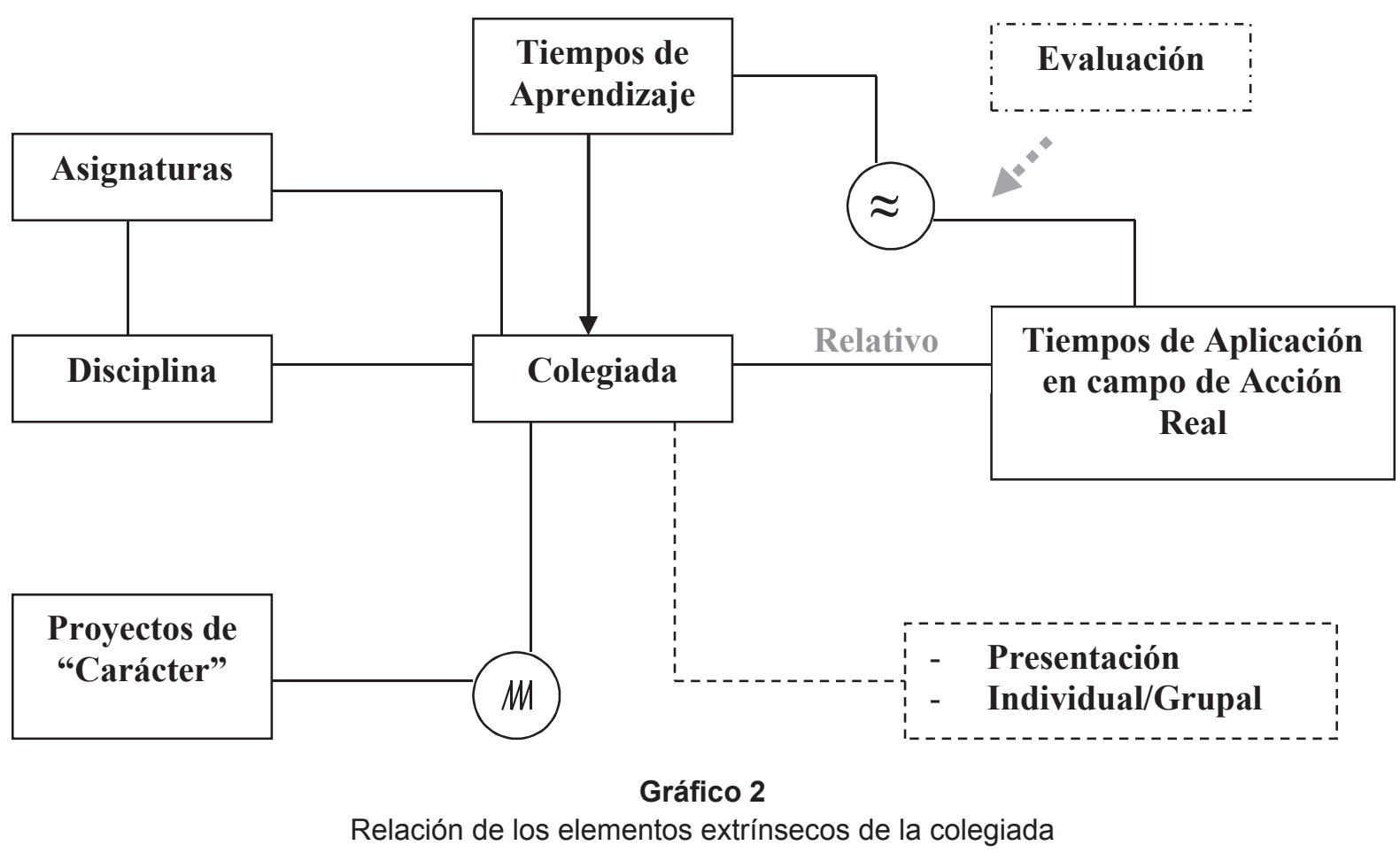


El método general -vox populi- de evaluación de proyectos consta de tres elementos básicos: [a] propuesta y análisis inicial; [b] seguimiento; y [c] evaluación y análisis final, siempre con el parámetro de realimentación y análisis constante ${ }^{7}$.

A la luz de esto, hemos de hablar de cuatro etapas en el proceso, para el afianzamiento en la aprehensión de conocimiento. La primera etapa es el análisis de estado actual respecto a un proyecto a ser evaluado, en cuyo estadio se presentan los elementos de proyecto, por cuanto se da un tiempo de asimilación de conocimientos de inicio para la presentación del proyecto; de esta manera se logran dos cosas: incentivar el autoaprendizaje y medir los niveles cognitivos iniciales. No es innecesario decir que durante todo el proceso, el aprendizaje presencial y asesoramiento es constante.

La segunda etapa, entendida como subdivisión dependiendo de los criterios pedagógicos, es de seguimiento del proyecto, durante el cual se hacen ajustes (si son necesarios) y potencializar el desarrollo del proyecto; el proceso de seguimiento es coadyuvado con investigación propia y el proceso presencial, el análisis de esta etapa permitirá determinar las herramientas necesarias y efectivas de aplicación por cuanto se ejecutan los presupuestos iniciales.

La tercera etapa hace referencia a la preevaluación o preentrega; ésta es la entrega total del proyecto con requerimientos académicos e implicaciones relativas, para ello la existencia o no de jueces de carácter objetivo (desvinculado del proceso) se encuentra subordinado a los deseos de la dirección del proyecto. No obs-

7 Baladí profundizar en dichos elementos sin la debida aplicación a la colegiada, y al proceso pedagógico llevado acabo. tante, es importante la evaluación con jurados subjetivos (vinculados al proceso) que permitan la evaluación de proceso, huelga recalcar la objetividad académica que en esta etapa acontece. Por medio de esto se logra el reconocimiento del nivel de afianzamiento de los conocimientos y parámetros de evaluación y análisis de los procesos pedagógicos y a su vez se presenta como una primera fase de determinación de diferencias entre la academia y el campo real de acción de conocimientos.

La cuarta etapa de "venta de proyecto" representa una emulación de presentación en campo real de acción, para lo cual se hace necesario la evaluación por parte de jurados objetivos (desvinculados del proceso) que permitirá un primer acercamiento de relación con al campo laboral que ejercita al estudiante desde la academia hacia el mercado.

Al contemplar la presentación como elemento compositivo, permite determinar los niveles en los cuales el proyecto es necesario ser alcanzado, ejemplo de ello es: teórico, bidimensional o tridimensional, simulado o prototipado, académico o laboral, entre otros, que deberán responder a las pertinencias singulares de cada proyecto, a su vez que se tendrá en cuenta la participación de una o varias personas por proyecto, así se tendrá claridad en los principios evaluativos.

Hasta el momento se han determinados los elementos constitutivos de la colegiada, mas la cantidad de ellas en un periodo lectivo se debe contemplar de acuerdo a la equidad entre tres parámetros: el académico, el institucional y el laboral. Por los cuales un proyecto cuenta con tres tiempos, que en general son disímiles; sin embargo, como lo pretendido es la preparación para el enfrentamiento a un mundo competitivo y mercadotecnista se requiere que los mayores valores de equidad deben ser el académico y 
el laboral, coadyuvados por la flexibilización administrativa institucional en dicho proceso, esto lleva a reconocer que dependiendo de las características singulares y generales de cada proyecto determinarán la cantidad de colegiadas en un periodo lectivo, que articulándolo con las reflexiones precedentes deben responder la fórmula:

$$
\mathrm{X}=1+\mathbf{n} \frac{\mathbf{t}_{\mathrm{e}}}{\mathbf{0 . 2} * \sum_{\mathbf{t}_{\mathrm{p}}}}
$$

Gráfico 3

Fórmula número de colegiadas por semestre

Donde " $x$ " es el número de colegiadas, " 1 " es la constante mínima de presentación entendida como formulación y seguimiento y evaluación, " $\mathrm{n}$ " es el número de temáticas pro periodo lectivo, " $t_{\mathrm{e}}$ " es el tiempo en días de duración de proyectos propuestos, y " 0.2 " es el margen de error contemplado en los tiempos de proyecto debido a la complejidad de determinar la equidad entre el aspecto académico y el laboral.

Al asumir la existencia de la colegiada no excluye el desarrollo de proyectos tanto a nivel intra-asignatura como interdisciplinares a lo largo del proceso, para ello se debe tener en cuenta que éstos son desarrollados para el fortalecimiento de subtemáticas con factor común en la baja complejidad, sean o no de relación directa con los proyectos de colegiada. No obstante, la restricción a un número limitado de ejemplificación que da la colegiada, hace recomendable la presentación de los subproyectos como elementos ejemplificantes de las diversas variantes de una misma temática y su 76
Por medio de la aplicación y análisis de las colegiadas se puede mensurar la pertinencia de las asignaturas y sus temáticas en el plan curricular.

\section{Propuesta para la evaluación ACADÉMICA DE LOS PROYECTOS DE DISEÑO EN LOS TALLERES DE DISEÑO}

Con base en la malla de congruencias entre los objetivos misionales institucionales, del programa de diseño y de los microcurrículos, sumados a las finalidades de los proyectos de taller en respuesta a las temáticas establecidas, a su vez que se articuló con reflexiones críticas, entrevistas y referentes bibliográfico, se determinaron los criterios más comunes e importantes para la evaluación de un proyecto de diseño. Dichos criterios permitirán tener claro tanto para los estudiantes como para los docentes, durante cada uno de los momentos evaluativos, los parámetros conceptuales que deberán ser cumplidos sean de manera teórica o práctica para el logro de los objetivos tanto del proyecto como de la asignatura. De igual manera, estos criterios, con base en las herramientas estadísticas, permiten un seguimiento de los procesos y los resultados para el mejoramiento continuo de la asignatura.

\subsection{Criterios de evaluación}

Los criterios de evaluación son categorizados bajo los grupos: académico, humano, empresarial y normativo. El académico hace referencia a los criterios vinculados directamente con las temáticas y conocimientos requeridos en la asignatura como fortalecimiento de la disciplina en órdenes del proceso de aprendizaje-enseñanza con carácter del saber-saber y el saber-hacer. El grupo humano contempla los criterios de evaluación que articula los comportamientos psicosociales de los estudiantes, en los cuales se involucran los elementos del saber-ser. El grupo empresarial compromete los criterios de evaluación que tienen relación con el contexto real de mercado, aunque la dinámica es una emulación de comportamiento frente a un cliente o usuario. El grupo normativo contiene los criterios de evaluación concernientes a aquellos parámetros establecidos para el cumplimiento de puesta en escena o entrega final del proyecto. 
Tabla 1

Criterios generales de evaluación

\begin{tabular}{|c|c|}
\hline \multirow{10}{*}{ 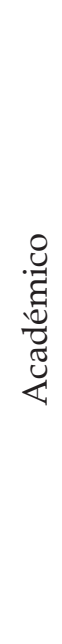 } & Acabados de Modelos \\
\hline & Concepto y Aplicación del Diseño \\
\hline & Aplicación de Recursos (Recursividad) \\
\hline & Aplicación Temática \\
\hline & Aplicación de Componentes Sistémicos \\
\hline & Informe \\
\hline & Relación Propuesta / Contexto \\
\hline & Comunicación Bi y Tridimensional \\
\hline & Investigación (Consulta Previa) \\
\hline & Aplicación Metodológica \\
\hline
\end{tabular}

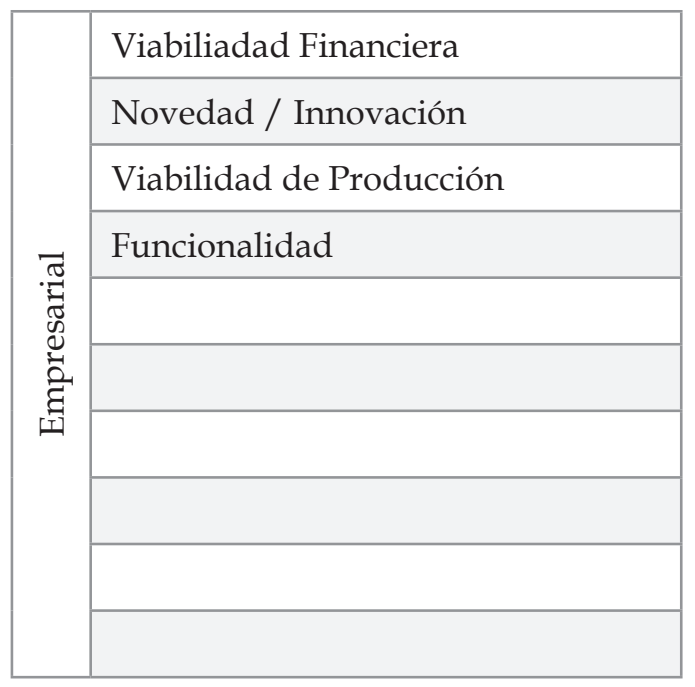

\begin{tabular}{|l|l|}
\hline \multirow{2}{*}{} & Presentación Personal \\
\cline { 2 - 3 } & Niveles de Cooperación \\
\cline { 2 - 3 } & Tiempos de Respuesta \\
\cline { 2 - 3 } & Niveles de AutoCensura (Ética) \\
\cline { 2 - 3 } & RetroAlimentación (Asesoría y Seguimiento) \\
\cline { 2 - 3 } & Roles y Jerarquías \\
\hline & Argumentación \\
\hline
\end{tabular}

\begin{tabular}{|c|c|}
\hline \multirow{7}{*}{ 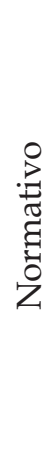 } & Número de Piezas \\
\hline & Presentación (Entrega) \\
\hline & Puntualidad \\
\hline & \\
\hline & \\
\hline & \\
\hline & \\
\hline
\end{tabular}

\subsection{Herramientas para evaluación}

Al mismo tiempo de la determinación de los criterios de evaluación se desarrollaron dos herramientas que permitirían tanto la cualificación y cuantificación de los procesos y los momentos evaluativos, como la consolidación de una estrategia de seguimiento para mejorar de manera continua los modelos y pedagogías usadas en cada una de las asignaturas, que se reflejan en los proyectos de diseño. Para la creación de estas herramientas se utilizó el programa especializado en tablas de cálculos Microsoft Excel® que permitió el uso de distribución y creación de fórmulas necesarias, en especial en la tabla de ponderación (ver tabla 3).

\subsubsection{Tabla de Evaluación in-Situ}

Esta tabla (ver tabla 2.) permite la recopilación, clasificación y evaluación de los proyectos por parte de los evaluadores y de los estudiantes durante el momento evaluativo conocido como entrega final. Los evaluadores diligenciarán los espacios destinados para la evaluación, previa ponderación porcentual de las características. Los estudiantes usarán la tabla como herramienta de autoevaluación a partir de los mismos criterios con los cuales serán evaluados; esto lleva a que los estudiantes tengan conocimiento previo de los criterios de evaluación y su valoración dentro del proyecto, a su vez que hagan un seguimiento de sus propios procesos en busca de la autogestión en calidad. 
Tabla 2

Tabla de evaluación de evaluadores y autoevaluación in situ

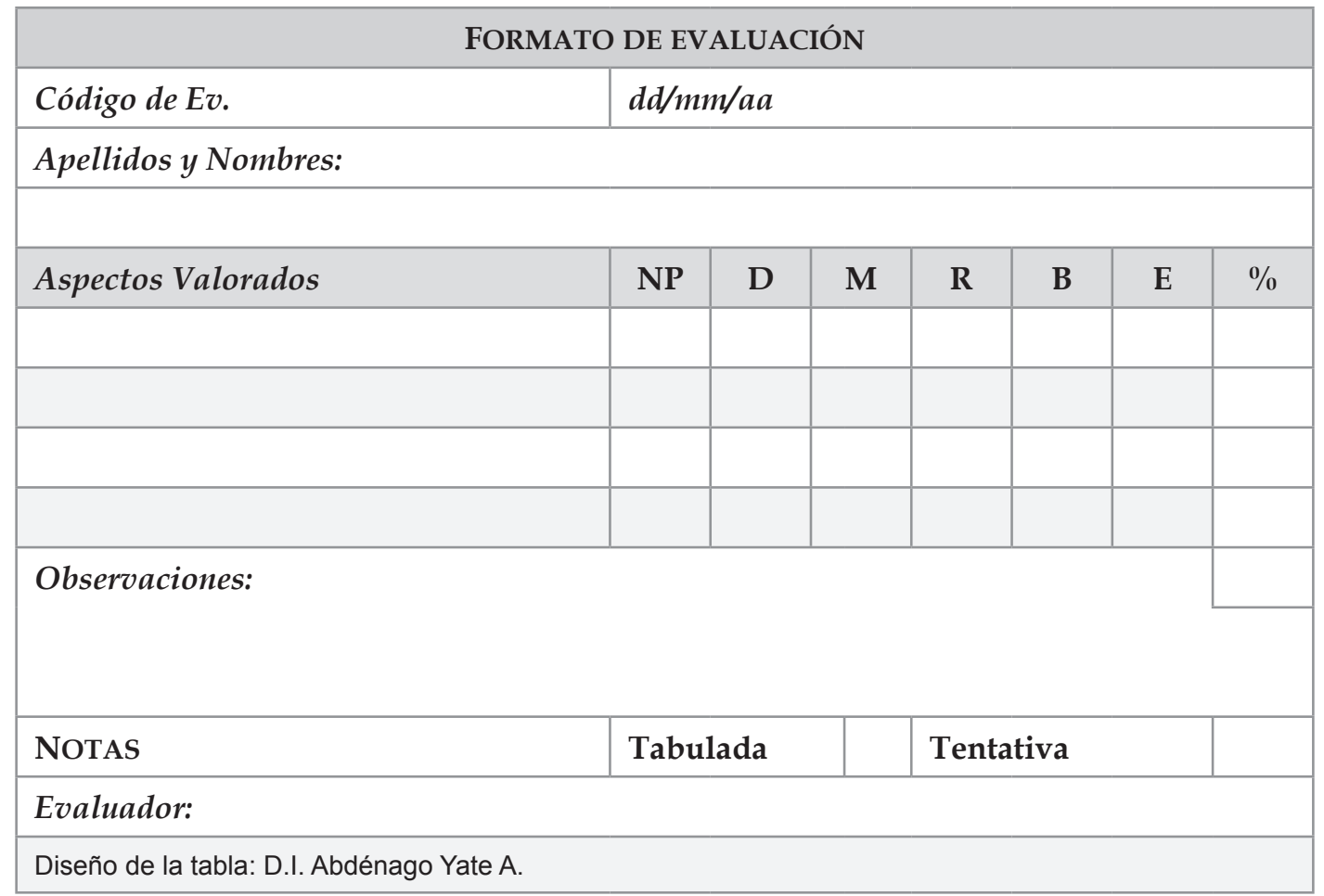

Esta tabla de evaluación in situ tiene el doble sentido de lograr por medio de una hoja de cálculo convertirse en una tabla de datos estadísticos que permitirá encontrar elementos comparativos para el mejoramiento continuo; como se presenta en el aparte de este artículo: Pruebas de campo y resultados.

A continuación se presenta la descripción de la tabla de evaluación in situ, y su modo de uso ${ }^{8}$.

1. Código de Ev. (Código de Evaluación): Para llevar un registro control de las evaluaciones realizadas cada proyecto tendrá un código que lo identifique; será dependiendo de la forma de registrar de cada institución.

78
2. DD-MM-AA: Corresponde al día, mes y año en que se llevó a cabo la evaluación.

$8 \quad$ Para ver la ejemplificación de diligenciamiento y uso ver el aparte: Pruebas de campo y resultados.
3. Apellido y Nombre: Corresponde a los Apellidos y los Nombres de los estudiantes evaluados por proyecto.

Los siguientes elementos corresponden a los criterios y ponderación de los datos obtenidos a partir de la Tabla de Evaluación.

4. Aspectos Valorados: son los criterios que han sido evaluados en la tabla de evaluación, ellos son los que la institución considere convenientes y aplicables a cada proyecto y correspondiente a cada nivel.

5. ED: Error Docente; corresponde a aspectos valorados que el docente/jurado evaluador no haya diligenciado en la tabla de evaluación; el porcentaje que corresponda a este aspecto valorado, se repartirá en el resto de aspectos valorados, por cuanto no se afecta negativamente al estudiante por un error docente. Vale la pena aclarar 
la inexistencia de un NO APLICA, debido a que todos los aspectos que se escojan para evaluar el proyecto deberán aplicar, por cuanto el aspecto valorado no diligenciado corresponde a Error Docente (ED). La tabla de Ponderación está diseñado para diligenciar estos espacios con el número uno (1) como equivalente a una equis $(\mathrm{x})$.

6. NP: No Presenta; corresponde a los aspectos valorados diligenciados siempre $\mathrm{y}$ cuando el estudiante no presente ningún indicador que permita medirlo. La tabla de Ponderación está diseñada para diligenciar estos espacios con el número uno (1) como equivalente a una equis (x).

7. $D, M, R, B, E$ : Corresponde respectivamente a Deficiente, Malo, Regular, Bueno y Excelente corresponde a las valoraciones dadas en cada uno de los aspectos valorados por parte del docente/ jurado evaluador. Se diligencia con una equis $(\mathrm{x})$.

8. \%: Porcentaje: es el porcentaje individual de cada uno de los aspectos valorados jerarquizando la importancia de cada uno de ellos dependiendo de las políticas institucionales y objetivos del proyecto evaluado. Este porcentaje debe hacerse en $100 \%$.

9. Notas Tabulada y Tentativa: como control y prueba valorativa y comparativa de evaluación existen dos espacios: Tentativa es el dato numérico que coloca el docente/ evaluador como si no usara el tabla, y Tabulada es el dato numérico obtenido a partir de la ponderación de los aspectos valorados y diligenciados por el docente/ evaluador.

Instrumentos de evaluación, seguimiento y ponderación de los trabajos de grado.

\subsubsection{Herramienta de ponderación de la evaluación}

La tabla de ponderación de la evaluación tiene como fin la conversión del diligenciamiento de la herramienta tabla de evaluación in situ; por medio de las fórmulas pertinentes se logra una conversión ágil, exacta y veraz de los valores cualitativos obtenidos en la tabla de evaluación in situ en valores numéricos requeridos para la validación académico administrativa sobre los conceptos de aprobado y reprobado.

Los elementos que componen la tabla de ponderación son:

Los tres primeros elementos corresponden a la identificación del proyecto y estudiantes evaluados. Sin embargo, si se desea éstos podrán ser obviados en la tabla de ponderación debido a que existen el registro en la tabla de evaluación.

1. Código de Ev. (Código de Evaluación): Para llevar un registro control de las evaluaciones realizadas cada proyecto tendrá un código que lo identifique; será dependiendo de la forma de registrar de cada institución.

2. DD-MM-AA: Corresponde al día, mes y año en que se llevó a cabo la evaluación.

3. Apellido y Nombre: Corresponde a los Apellidos y los Nombres de los estudiantes evaluados por proyecto.

Los siguientes elementos corresponden a los criterios y ponderación de los datos obtenidos a partir de la tabla de evaluación in situ; esto significa que la tabla de ponderación deberá ser diligenciada por cara tabla de evaluación.

4. Aspectos Valorados: son los criterios que han sido evaluados en la tabla de evaluación, ellos son los que la institución considere convenientes y aplicables a cada proyecto y correspondiente a cada nivel. 
Tabla 3

Tabla de ponderación de valores de evaluación. (Ejemplo de aplicación)

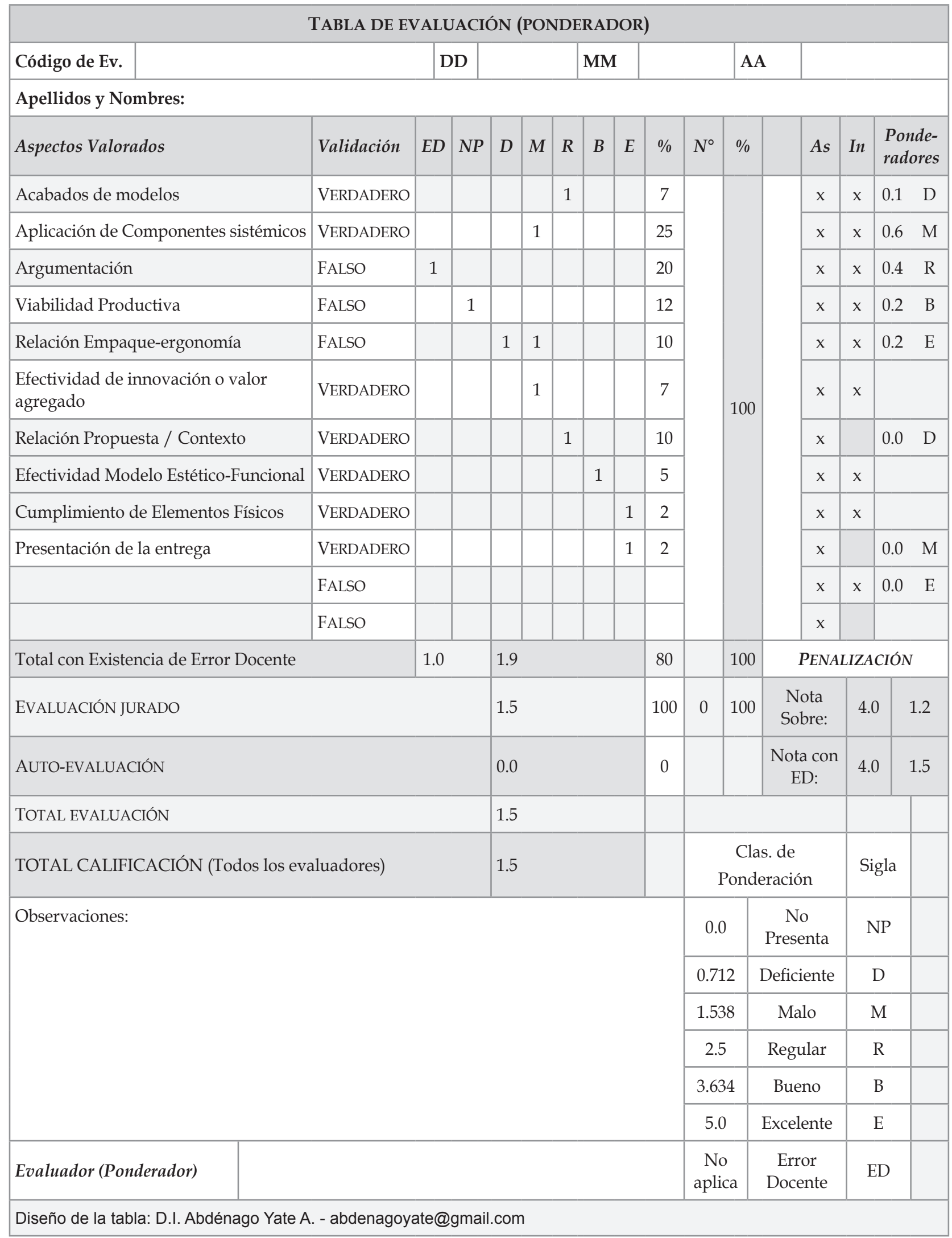


5. ED: Error Docente; corresponde a aspectos valorados que el docente/jurado evaluador no haya diligenciado en la tabla de evaluación; el porcentaje que corresponda a este aspecto valorado, se repartirá en el resto de aspectos valorados, por cuanto no se afecta negativamente al estudiante por un error docente. Vale la pena aclarar la inexistencia de un NO APLICA, debido a que todos los aspectos que se escojan para evaluar el proyecto deberán aplicar, por cuanto el aspecto valorado no diligenciado corresponde a Error Docente (ED). La tabla de Ponderación está diseñada para diligenciar estos espacios con el número uno (1) como equivalente a una equis $(\mathrm{x})$.

6. NP: No Presenta; corresponde a los aspectos valorados diligenciados siempre y cuando el estudiante no presente ningún indicador que permita medirlo. La tabla de Ponderación está diseñada para diligenciar estos espacios con el número uno (1) como equivalente a una equis $(x)$.

7. $D, M, R, B, E$ : Corresponde respectivamente a Deficiente, Malo, Regular, Bueno y Excelente corresponde a las valoraciones dadas en cada uno de los aspectos valorados por parte del docente/jurado evaluador. La tabla de Ponderación está diseñada para diligenciar estos espacios con el número uno (1) como equivalente a una equis $(\mathrm{x})$.

8. Valoración: Hace referencia a controlar el buen diligenciamiento de la evaluación en tanto que registra VERDADERO cuando está bien diligenciado, y FALSO cuando esto no ocurra, como lo es: omisión y repetición de diligenciamiento de un aspecto valorado.

9. \%: Porcentaje; es el porcentaje individual de cada uno de los aspectos valorados jerarquizando la importancia de cada uno de ellos dependiendo de las políticas institucionales y objetivos del proyecto evaluado. Este porcentaje debe hacerse en $100 \%$.

10.Nov.: Novedad; cantidad de aspectos valorados.

11.Evaluación Jurado: Dato numérico de la ponderación de los datos del docente/ jurado evaluador. Este dato se hace sobre una nota de 5,0. Si existe una autoevaluación del evaluado, tanto el docente/ jurado y el evaluado tiene su propio porcentaje sobre el total de la evaluación.

12.Total con Existencia de Error Docente: Dato numérico de la ponderación de los datos del docente/jurado evaluador. Este dato se hace sobre una nota de 5,0, contemplando el error de diligenciamiento de la tabla de evaluación por parte del docente/jurado evaluador, como lo son la omisión o la repetición de evaluación de un mismo aspecto valorado.

13.Auto evaluación: Dato numérico de la evaluación dada por los evaluados, a partir de la tabla de evaluación destinada a ellos.

14.Total Evaluación: corresponde al dato numérico, contemplando que no exista ningún Error Docente.

15.Penalización: Dato numérico que corresponde a la evaluación, si existe una penalización a los evaluados por motivos determinados por la institución.

16. Clas. de Ponderación: Clasificación de la Ponderación; corresponde a los datos numéricos de evaluación ED, NP, D, M, R, B y E.

Los datos que se presentan corresponden a una función derivada a partir de una curva de dificultad, que permite medir el paso de un nivel al otro. La curva es: 


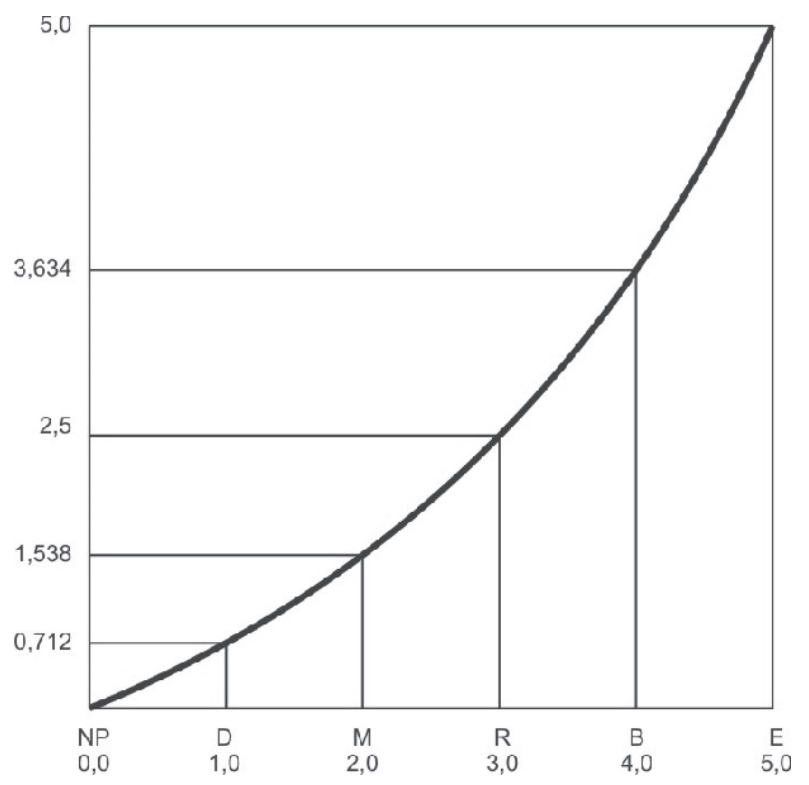

Gráfico 4

Curva de datos numéricos de clasificación de ponderación

Estos datos de ponderación han salido a partir de las reflexiones teórico-prácticas referidas a los niveles de complejidad al superar cada una de las etapas del proyecto, lo que se refleja en los diferentes niveles de características cualitativas; en otras palabras, la dificultad de pasar en cada un de los grados no presenta, deficiente, malo, regular, bueno y excelente.

\section{Pruebas de campo y Resultados}

A continuación se presentan las tablas correspondientes a siete pruebas pilotos desarrolladas desde el 2007 hasta el 2011 en las cuales se puso a prueba el modelo de evaluación y sus respectivas herramientas. Estas pruebas se 1levaron a cabo en los distintos talleres de diseño y de empaques en el área proyectual del programa de diseño industrial de la Universidad Autónoma de Colombia.

Primera prueba piloto: Datos generales: Asignatura Taller de Diseño 3: Biónica (28/11/2007) 13 grupos evaluados (32 estudiantes en total) - 2 evaluadores.

Tabla 4

Tabla de evaluación in situ diligenciada y convertida en herramienta estadística.

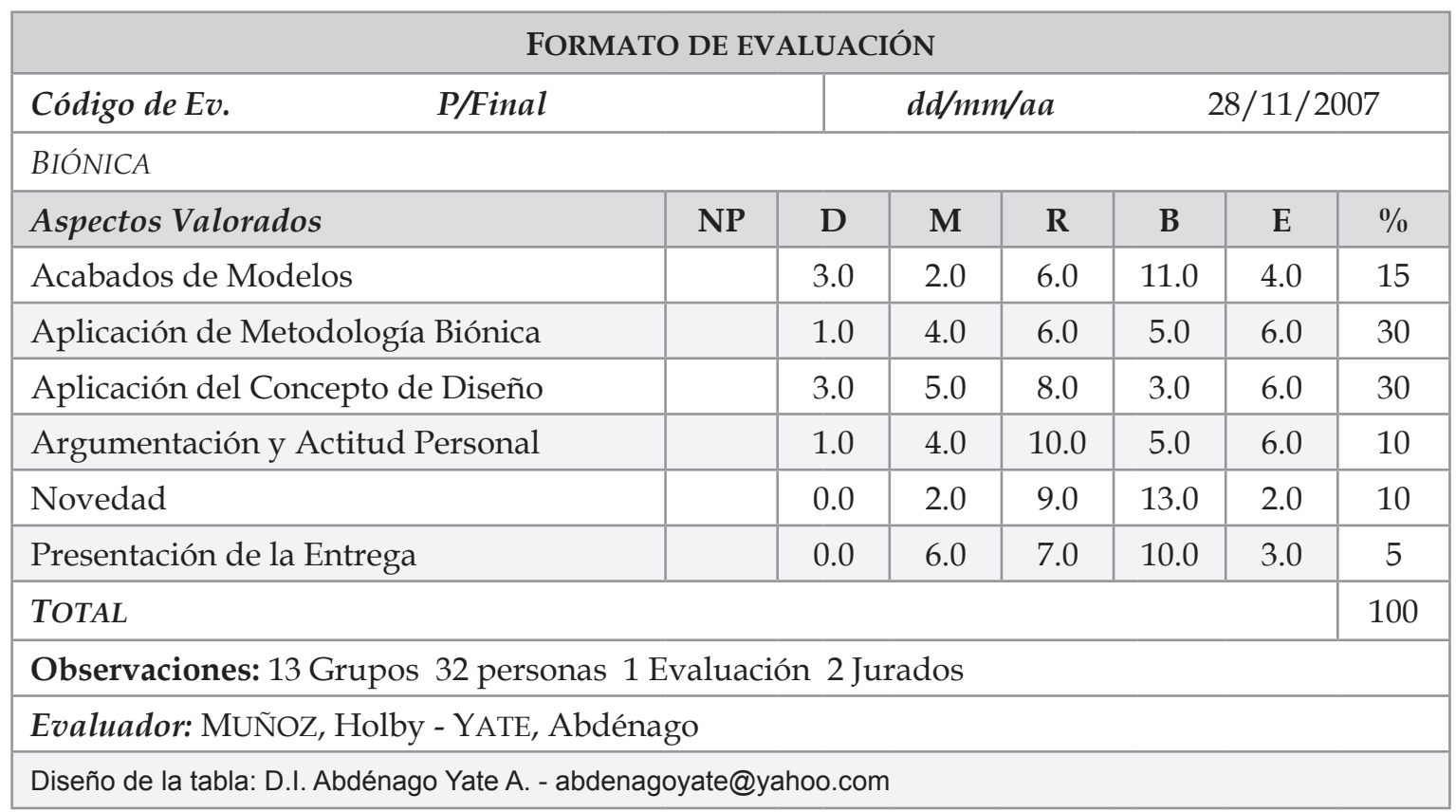

Segunda prueba piloto: Datos generales: Asignatura Taller de Diseño 3: Biónica (22/05/2008) -
17 grupos evaluados (36 estudiantes en total) - 2 evaluadores. 
Tabla 5

Tabla de evaluación in situ diligenciada y convertida en herramienta estadística.

\begin{tabular}{|c|c|c|c|c|c|c|c|}
\hline \multicolumn{8}{|c|}{ FORMATO DE EVALUACIÓN (jurado Principal) } \\
\hline \multicolumn{3}{|c|}{ Código de Ev.: Bandeja Paisa } & \multicolumn{2}{|c|}{$d d / m m / a a$} & \multicolumn{2}{|c|}{$22 / 05 / 2008$} & \\
\hline \multicolumn{8}{|l|}{ BIÓNICA } \\
\hline Aspectos Valorados & NP & D & $\mathbf{M}$ & $\mathbf{R}$ & B & $\mathrm{E}$ & $\%$ \\
\hline Acabados de Modelos & 1.0 & & 3.0 & 9.0 & 19.0 & 4.0 & 15 \\
\hline Investigación (Consulta Previa) & & & 1.0 & 7.0 & 23.0 & 5.0 & 10 \\
\hline Aplicación del Concepto de Diseño & & & 1.0 & 19.0 & 13.0 & 3.0 & 10 \\
\hline $\begin{array}{l}\text { Secuencia de Uso / funcionalidad/ } \\
\text { Ergonomía/antropometría }\end{array}$ & 2.0 & & 10.0 & 12.0 & 8.0 & 4.0 & 15 \\
\hline Relación Sistémica/Familiar. & & 1.0 & 3.0 & 13.0 & 9.0 & 9.0 & 12 \\
\hline Novedad/Innovación & & & 10.0 & 15.0 & 7.0 & 4.0 & 5 \\
\hline Aplicación de Método Biónico & & & 5.0 & 11.0 & 19.0 & 1.0 & 20 \\
\hline Claridad de síntesis información/Argum. & & & 2.0 & 16.0 & 12.0 & 5.0 & 5 \\
\hline Comunicación bidimensional & & & 1.0 & 14.0 & 15.0 & 5.0 & 5 \\
\hline $\begin{array}{l}\text { Presentación de la Entrega/Personal/ } \\
\text { Actitud Personal }\end{array}$ & & & & 11.0 & 24.0 & 1.0 & 3 \\
\hline \multicolumn{7}{|l|}{ TOTAL } & 100 \\
\hline \multicolumn{8}{|c|}{ Observaciones: 17 Grupos 36 personas 1 Evaluación 2 Jurados } \\
\hline \multicolumn{8}{|c|}{ Evaluadores: YATE, Abdénago; AGUDELO, Isabel Cristina } \\
\hline \multicolumn{8}{|c|}{ Diseño de la tabla: D.I. Abdénago Yate A. } \\
\hline
\end{tabular}

Tercera prueba piloto: Datos generales: Asignatura Taller de Empaques 2 (04/03/2008) - 12 grupos evaluados (21 estudiantes en total) - 1 evaluador.

Tabla 6

Tabla de evaluación in situ diligenciada y convertida en herramienta estadística

\begin{tabular}{|c|c|c|c|c|c|c|c|}
\hline \multicolumn{8}{|c|}{ FORMATO DE EVALUACIÓN } \\
\hline \multicolumn{2}{|c|}{ Código de Ev. P. Contexto RitoCafé } & \multicolumn{3}{|c|}{$d d / m m / a a$} & \multicolumn{2}{|c|}{$04 / 03 / 2008$} & \\
\hline \multicolumn{8}{|l|}{ Taller de Empaques 2} \\
\hline Aspectos Valorados & NP & D & $\mathbf{M}$ & $\mathbf{R}$ & B & $\mathbf{E}$ & $\%$ \\
\hline Acabados de Modelos & & 0.0 & 1.0 & 4.0 & 5.0 & 2.0 & 10 \\
\hline Investigación (Consulta Previa) & & 0.0 & 0.0 & 3.0 & 8.0 & 1.0 & 5 \\
\hline Aplicación del Concepto de Diseño & & 0.0 & 0.0 & 1.0 & 9.0 & 2.0 & 25 \\
\hline Comunicación Bi y Tridimensional & & 0.0 & 2.0 & 3.0 & 6.0 & 1.0 & 15 \\
\hline Aplicación Componentes Sistémicos & & 0.0 & 1.0 & 6.0 & 4.0 & 1.0 & 5 \\
\hline Novedad/Innovación & & 1.0 & 3.0 & 3.0 & 4.0 & 1.0 & 8 \\
\hline Relación Propuesta/Contexto & & 0.0 & 0.0 & 1.0 & 8.0 & 3.0 & 20 \\
\hline Presentación Personal & & 0.0 & 2.0 & 7.0 & 2.0 & 1.0 & 3 \\
\hline Argumentación y Actitud Personal & & 0.0 & 0.0 & 1.0 & 10.0 & 1.0 & 5 \\
\hline Presentación de la Entrega & & 1.0 & 1.0 & 5.0 & 3.0 & 2.0 & 4 \\
\hline \multicolumn{7}{|l|}{ TOTAL } & 100 \\
\hline \multicolumn{8}{|c|}{ Observaciones: 12 Grupos 21 personas 1 Evaluación 1 Jurados } \\
\hline \multicolumn{8}{|l|}{ Evaluador: YATE, Abdénago } \\
\hline \multicolumn{8}{|l|}{ Diseño de la tabla: D.I. Abdénago Yate A. } \\
\hline
\end{tabular}


Cuarta prueba piloto: Datos generales: Asigna- grupos evaluados (19 estudiantes en total) tura Taller de Empaques 2 (28/02/2008) - 111 evaluador.

Tabla 7

Tabla de evaluación in situ diligenciada y convertida en herramienta estadística

\begin{tabular}{|c|c|c|c|c|c|c|c|}
\hline \multicolumn{8}{|c|}{ FORMATO DE EVALUACIÓN } \\
\hline \multicolumn{3}{|c|}{ Código de Ev. P. Mat. No Convencio. } & \multicolumn{2}{|c|}{$d d / m m / a a$} & \multicolumn{2}{|c|}{$28 / 02 / 2008$} & \\
\hline \multicolumn{8}{|l|}{ Taller de Empaques 2} \\
\hline Aspectos Valorados & NP & D & $\mathbf{M}$ & $\mathbf{R}$ & B & $\mathrm{E}$ & $\%$ \\
\hline Acabados de Modelos & & 0.0 & 0.0 & 2.0 & 1.0 & 8.0 & 10 \\
\hline Investigación (Consulta Previa) & & 0.0 & 0.0 & 1.0 & 5.0 & 5.0 & 10 \\
\hline Aplicación del Concepto de Diseño & & 0.0 & 0.0 & 3.0 & 8.0 & 0.0 & 25 \\
\hline Aplicación Concepto Cultural & & 0.0 & 0.0 & 2.0 & 8.0 & 1.0 & 20 \\
\hline Aplicación Componentes Sistémicos & & 0.0 & 1.0 & 6.0 & 3.0 & 1.0 & 10 \\
\hline Novedad/Innovación & & 1.0 & 1.0 & 4.0 & 3.0 & 2.0 & 8 \\
\hline Viabilidad de Producción & & 0.0 & 0.0 & 0.0 & 2.0 & 9.0 & 5 \\
\hline Presentación Personal & & 0.0 & 1.0 & 4.0 & 4.0 & 2.0 & 3 \\
\hline Argumentación y Actitud Personal & & 0.0 & 0.0 & 1.0 & 7.0 & 3.0 & 5 \\
\hline Presentación de la Entrega & & 0.0 & 2.0 & 2.0 & 6.0 & 1.0 & 4 \\
\hline \multicolumn{7}{|l|}{ TOTAL } & 100 \\
\hline \multicolumn{8}{|c|}{ Observaciones: 11 Grupos 19 personas 1 Evaluación 1 Jurados } \\
\hline \multicolumn{8}{|c|}{ Evaluador: YATE, Abdénago } \\
\hline \multicolumn{8}{|l|}{ Diseño de la tabla: D.I. Abdénago Yate A. } \\
\hline
\end{tabular}

Quinta prueba piloto: Datos generales: Asigna- grupos evaluados (20 estudiantes en total) - 1 tura Taller de Empaques $2(06 / 03 / 2008)-1$ evaluador.

Tabla 8

Tabla de evaluación in situ diligenciada y convertida en herramienta estadística.

\begin{tabular}{|c|c|c|c|c|c|c|c|}
\hline \multicolumn{8}{|c|}{ FORMATO DE EVALUACIÓN } \\
\hline \multicolumn{3}{|l|}{ Código de Ev. P/Frutas/Sec. De Uso } & \multicolumn{2}{|c|}{$d d / m m / a a$} & \multicolumn{3}{|c|}{$06 / 03 / 2008$} \\
\hline \multicolumn{8}{|l|}{ Taller de Empaques 2} \\
\hline Aspectos Valorados & NP & D & $\mathbf{M}$ & $\mathbf{R}$ & B & E & $\%$ \\
\hline Acabados de Modelos & & 0.0 & 1.0 & 1.0 & 4.0 & 5.0 & 10 \\
\hline Investigación (Consulta Previa) & & 0.0 & 0.0 & 0.0 & 0.0 & 11.0 & 5 \\
\hline Aplicación del Concepto de Diseño & & 0.0 & 0.0 & 0.0 & 6.0 & 5.0 & 20 \\
\hline Aplicación Concepto Cultural & & 0.0 & 0.0 & 0.0 & 7.0 & 4.0 & 15 \\
\hline Aplicación Componentes Sistémicos & & 0.0 & 0.0 & 0.0 & 1.0 & 10.0 & 10 \\
\hline Efecti. Didác. Secuencia de Uso & & 0.0 & 0.0 & 0.0 & 3.0 & 9.0 & 18 \\
\hline Viabilidad de Producción & & 0.0 & 0.0 & 0.0 & 1.0 & 10.0 & 10 \\
\hline Presentación Personal y Act. Personal & & 0.0 & 0.0 & 3.0 & 2.0 & 6.0 & 3 \\
\hline Argumentación & & 0.0 & 0.0 & 1.0 & 3.0 & 7.0 & 5 \\
\hline Presentación de la Entrega & & 0.0 & 0.0 & 0.0 & 8.0 & 3.0 & 4 \\
\hline \multicolumn{7}{|l|}{ TOTAL } & 100 \\
\hline \multicolumn{8}{|c|}{ Observaciones: 11 Grupos 20 personas 1 Evaluación 1 Jurados } \\
\hline \multirow{2}{*}{\multicolumn{8}{|c|}{ Evaluador: YATE, Abdénago }} \\
\hline & & & & & & & Diseño de la tabla: D.I. Abdénago Yate A. \\
\hline
\end{tabular}


Sexta prueba piloto: Datos generales: Asignatura Taller de Diseño 5: Contexto (03/12/2007) - 14 grupos evaluados (46 estudiantes en total) - 2 evaluadores.

\begin{tabular}{|c|c|c|c|c|c|c|c|c|c|}
\hline \multicolumn{10}{|c|}{ FORMATO DE EVALUACIÓN } \\
\hline \multicolumn{3}{|l|}{ Código de Ev.: Grupo J.C.S. } & \multicolumn{2}{|l|}{ dd } & 3 & $\mathbf{m m}$ & 12 & aa & 2007 \\
\hline \multicolumn{10}{|l|}{ CONTEXTO (Diseño 5) } \\
\hline Aspectos Valorados & NP & $\mathbf{D}$ & $\mathbf{M}$ & $\mathbf{R}$ & B & E & $\%$ & N. & $\%$ \\
\hline Comunicación Bi y Tridimensional & & 1.0 & 3.0 & 1.0 & 9.0 & 2.0 & 30 & \multirow{3}{*}{3} & \multirow{3}{*}{100} \\
\hline Innovación y Concepto de Diseño & & & 6.0 & 3.0 & 7.0 & 2.0 & 30 & & \\
\hline Relación Propuesta-Contexto & & & 5.0 & 7.0 & 5.0 & 1.0 & 40 & & \\
\hline \multicolumn{7}{|l|}{ TOTAL } & 100 & 3 & 100 \\
\hline \multicolumn{10}{|c|}{ Observaciones: 14 grupos - 46 estudiantes } \\
\hline \multicolumn{10}{|c|}{ Evaluador: Juan Carlos Silva - Abdénago Yate } \\
\hline \multicolumn{10}{|l|}{ Diseño de la tabla: D.I. Abdénago Yate A. } \\
\hline
\end{tabular}

\section{CONCLUSIONES Y SUGERENCIAS}

En las pruebas pilotos, en las que se contemplaron diferentes asignaturas, tipos de proyectos y criterios de evaluación, se hace evidente el gran potencial del modelo de evaluación desarrollado en la presente investigación debido a que permite que éste pueda ser aplicado en las a las distintas disciplinas de diseño, sean éstas industrial, gráfico, modas, multimedia, etcétera; ya que dichas disciplinas fundamentan sus modelos de aprendizaje enseñanza en la modalidad de proyecto como estrategia que fortalece la aprehensión del conocimiento a partir de dinámicas teórico-prácticas que emulan las diversas situaciones del ejercicio profesional en el sector empresarial a nivel de un mercado altamente competitivo. De igual manera, que permite el seguimiento de los procesos y resultados para el mejoramiento continuo, tanto de los estudiantes como de los docentes y en especial la asignatura. En este sentido, el modelo se consolida más allá de una estrategia con sus respectivas herramientas para el fortalecimiento disciplinar desde los currículos, sino que por medio de las herramientas y resultados estadísticos coadyuvan en un entendimiento de cada una de las variables que configuran los proyectos, los microcurrículos y los currículos, conllevando un registro que sirve de soporte para futuros procesos, que tengan como objetivo la calidad de la educación en las disciplinas que tengan al proyecto como una modalidad de aprendizaje-enseñanza.

Por otro lado, es de notar que el modelo cuenta con herramientas tecnológicas que facilitan la evaluación en un momento específico concreto, cuyo precedente es una juiciosa, acuciosa y detallada preparación. De aquí se desprende el impacto en la comunidad docente y estudiantil sobre las dinámicas de evaluación como parte del proceso de aprendizaje-enseñanza y no meramente un momento en el cual se obtiene una calificación numérica para aprobar o reprobar. Es así que coadyuva en la concienciación del valor y la importancia de la evaluación dentro de las disciplinas de diseño y por ende su profesional preparación, desarrollo, aplicación, resultados y seguimiento.

Por medio de las pruebas se comprobó uno de los objetivos de la investigación en cuanto al desarrollo un modelo de evaluación que per- 
mita la claridad a los estudiantes, y docentes, de los criterios de evaluación. La claridad de los criterios de evaluación dados en el comienzo de cada proyecto permitió a los estudiantes posicionarlos como objetivos de sus procesos en tanto que se convierten en una fortaleza en la aprehensión del conocimiento, sumado a que ellos hacen parte de su momento evaluativo como actores activos se logró una reducción a cero los reclamos que antes de aplicar el modelo se presentaban por la falta de claridad en el origen de las calificaciones numéricas.

\section{Referencias}

Bürdek, Bernhard E. (1994). Diseño. Historia, Teoría y Práctica del Diseño Industrial. Editorial Gustavo Gili, S.L. Barcelona, España. ISBN: 8425216192. ISBN-13: 9788425216190.

ICSID (2009). International council of societies of industrial design (27 de septiembre del 2009). About IDCI. History. Http:/ / www.icsid.org/about/about/articles33.htm.

Lesmes S., Luis Alberto (2009). Pautas para el diseño de contenidos digitales pedagógicos. Reflexiones sobre las metodologías para diseñar productos multimedia tomando como referencia la enseñanza del diseño industrial. Universidad Nacional de Colombia. Monografía de grado para optar al título de magíster en diseño de multimedia. Director José Eduardo Naranjo. 25 de junio de 2009.

Navarro Saldaña, Alfredo (2001). Introducción al concepto de diseño o cultura del proyecto en el mundo contemporáneo. En Lenguajes, comunicación y técnicas. Jornadas de Formación del Profesorado. Consejería de Educación del Gobierno de Cantabria, Santander.

RAE [1]. Real Academia de la Lengua Española. Diccionario de la Lengua Española - Vigésima segunda edición.

http:/ / buscon.rae.es/draeI/SrvltConsulta?TIPO_BUS=3\&LEMA=gr\%C3\% A1fico [30/09/2009 12:08 p.m. [2].

http:/ / buscon.rae.es/draeI/SrvltConsulta?TIPO_BUS=3\&LEMA=gr\%C3\% A1fico [30/09/2009 12:26 p.m.]

Uniandes (2009). Universidad de los Andes. Facultad de arquitectura y diseño. Departamento de diseño. Programa. http:// design.uniandes.edu.co/programa/ [02/08/2009 08:56:08 p.m.]

WIPO (2009). Organization mondiale de la propriété intellectuelle. OMPI. Dessins et modèles industriels. Activités.http://www.wipo.int/about-ip/es/industrial_designs.htmlResources http://www.wipo.int/sme/fr/ip_business/industrial_designs/index.htm

86 Yate A., Abdénago y Lesmes S., Luis Alberto (2010). Artículo: La evaluación educativa en diseño industrial. Revista Actas de Diseño $\mathrm{N}^{\circ}$ 8. IV Encuentro latinoamericano de diseño «Diseño en Palermo» Comunicaciones académicas Junio 2009. Foro de Escuelas de Diseño. Facultad de Diseño y Comunicación. Universidad de Palermo. Marzo 2010 Año 4, pp. 68-71. Buenos Aires, Argentina. ISSN: 1850 -2032. 